Pacific Journal of Mathematics

ON SEMIGROUPS OF CONVOLUTION OPERATORS IN 


\title{
ON SEMIGROUPS OF CONVOLUTION OPERATORS IN HILBERT SPACE
}

\author{
JORGE D. SAMUR
}

\begin{abstract}
Given an infinitely divisible probability measure on a real separable Hilbert space $H$ and the infinitesimal generator $A$ of the associated semigroup of convolution operators acting on the Banach space of bounded uniformly continuous real functions on $H$, we describe the action of $A$ on certain classes of differentiable functions.
\end{abstract}

1. Introduction. For every infinitely divisible probability measure $\mu$ on a real separable Banach space $E$ there is an associated strongly continuous semigroup of convolution operators on the Banach space $C_{u}(E)$, the class of bounded uniformly continuous real-valued functions on $E$ with the norm of uniform convergence. According to the general theory of semigroups of operators, the domain of the infinitesimal generator of every such semigroup is dense in $C_{u}(E)$. As is well known, one of the central aspects of the study of a specific semigroup of operators is the description of the action of its infinitesimal generator on a class of "smooth" functions which is large enough to characterize the semigroup. In the case when $E$ is finite-dimensional, a result of this kind was obtained by Courrège [3], where the action of all generators of convolution semigroups on a natural class of differentiable functions is described. When $E$ is an infinite-dimensional Banach space, however, the scarcity of differentiable functions (see $[*]$ for a recent discussion) does not allow such a description.

This difficulty can be surmounted in the case when $E$ is a Hilbert space; this is the object of the present paper. We consider the case in which $E$ is a Hilbert space $H$ and describe the action of the generators on certain classes of differentiable functions. We exhibit a natural class of differentiable functions - the class $C_{u}^{(2)}(H)$, defined below - on which all generators of convolution semigroups can be characterized (Theorem 3.1); our result generalizes the work of Courrège [3]. However, in contrast to the situation in the finite-dimensional case, $C_{u}^{(2)}(H)$ is not dense in $C_{u}(H)$ when $H$ is infinite-dimensional.

It is possible to prove a stronger result for convolution semigroups without Gaussian component; in fact, in Theorem 3.6 we describe the 
action of the generator of any such semigroup on a dense class of differentiable functions. Finally, an extension for the Hilbert case of a result of L. Gross ([4]) is stated (Corollary 3.7).

As a by-product, we obtain the well-known Lévy-Khintchine representation in Hilbert space including limit formulas for the terms of the representation which strengthen the known results.

At several points we use ideas and techniques of [2].

$H$ will denote a real separable Hilbert space with $\|\cdot\|=\langle\cdot, \cdot\rangle^{1 / 2}$; the norm of $C_{u}(H)$ (defined above) is also denoted $\|\cdot\|$. If $f: H \rightarrow \mathbf{R}$ is a continuous function which is Fréchet differentiable at $x \in H$ we denote by $f^{\prime}(x)$ the point of $H$ such that $\left\langle f^{\prime}(x), \cdot\right\rangle$ is the derivative of $f$ at $x$; analogously, $f^{\prime \prime}(x)$ will be the symmetric bounded operator on $H$ such that $\left\langle f^{\prime \prime}(x)(\cdot), \cdot\right\rangle$ is the second Fréchet derivative of $f$ at $x$, if it exists. $C_{u}^{(2)}(H)$ will be the subspace of $C_{u}(H)$ of those twice Fréchet differentiable functions $f$ such that

$$
\left\|f^{\prime}\right\|=\sup _{x \in H}\left\|f^{\prime}(x)\right\|<\infty, \quad\left\|f^{\prime \prime}\right\|=\sup _{x \in H}\left\|f^{\prime \prime}(x)\right\|<\infty
$$

and $f^{\prime \prime}$ is uniformly continuous (again, we write $\|\cdot\|$ for different norms).

All measures considered are defined on the Borel $\sigma$-algebra of $H$. We refer to [6] for the definition and properties of weak convergence, symbolized here by $\stackrel{w}{\rightarrow} . N$ denotes the set of natural numbers greater than zero.

2. Semigroups of measures. A probability measure $\mu$ on a separable Banach space $E$ is infinitely divisible if for each $n \in N$ there exists a probability measure $\mu_{1 / n}$ on $E$ such that $\left(\mu_{1 / n}\right)^{n}=\mu\left(\nu^{n}\right.$ denotes the $n$th convolution power of a finite measure $\nu$ ). The characteristic functional $\hat{\mu}$ of $\mu$ never vanishes on $E^{\prime}$ (the topological dual of $E$ ) and, consequently (see [2], §2), there exists a unique sequentially $w^{*}$-continuous function $l$ : $E^{\prime} \rightarrow \mathbf{C}$ such that $\hat{\mu}=\exp l$ and $l(0)=0$. The $n$th root of $\mu$ is unique and $\hat{\mu}_{1 / n}=\exp ((1 / n) l)$.

Recall that a family $\left\{\mu_{t}: t \geq 0\right\}$ of probability measures on $E$ is a (weakly continuous) semigroup of measures if (1) $\mu_{0}=\delta_{0}$, (2) $\mu_{s} * \mu_{t}=\mu_{s+t}$ for $s \geq 0, t \geq 0$, and (3) $\mu_{t} \stackrel{w}{\rightarrow} \mu_{0}$ as $t \rightarrow 0$.

The following fact is well-known; we sketch a proof for the sake of completeness.

Proposition 2.1. For every infinitely divisible probability measure $\mu$ on a separable Banach space $E$ there exists a unique semigroup of measures $\left\{\mu_{t}: t \geq 0\right\}$ such that $\mu_{1}=\mu$. 
Sketch of proof. We outline only the construction of $\left\{\mu_{t}\right\}$. The existence of $l: E^{\prime} \rightarrow \mathbf{C}$ as above such that $\hat{\mu}=\exp l$ implies that for a positive rational $r=k / n(k, n \in N)$ we may define $\mu_{r}=\left(\mu_{1 / n}\right)^{k}$ depending only on $r$; let $\mu_{0}=\delta_{0}$. We have now a semigroup $\left\{\mu_{r}\right\}$ with non-negative rational parameter such that $\mu_{1}=\mu$.

In order to define $\mu_{t}$ for real positive $t$, observe that if $\left\{r_{n}\right\}$ is a bounded sequence of positive rationals the semigroup property gives that $\left\{\mu_{r_{n}}\right\}$ is relatively shift compact (see Def. 2.1 and Th. 2.2 of Ch. III in [6]) and recall the following result: if $\left\{\nu_{n}\right\}$ is a sequence of probability measures on $E$ such that (1) $\left\{\nu_{n}\right\}$ is relatively shift compact and (2) $\left\{\hat{\nu}_{n}\right\}$ converges uniformly on the balls of $E^{\prime}$ to a certain function $g$, then there exists a probability measure $\nu$ on $E$ such that $\nu_{n} \stackrel{w}{\rightarrow} v$ and $\hat{v}=g$ (this is proved as Th. 4.5, Ch. VI in [6]).

Given a semigroup of measures $\left\{\mu_{t}: t \geq 0\right\}$ on $H$, for each $t>0$ we define the point $x_{t} \in H$, the bounded symmetric operator $T_{t}$ on $H$ and the finite positive measure $\nu_{t}$ on $H$ by

$$
\begin{aligned}
x_{t} & =\int \frac{x}{1+\|x\|^{2}}\left(\frac{1}{t}\right) \mu_{t}(d x), \\
\left\langle T_{t} y, y\right\rangle & =\int \frac{\langle x, y\rangle^{2}}{\left(1+\|x\|^{2}\right)^{2}}\left(\frac{1}{t}\right) \mu_{t}(d x) \quad(y \in H)
\end{aligned}
$$

and

$$
\nu_{t}(d x)=\frac{\|x\|^{2}}{1+\|x\|^{2}}\left(\frac{1}{t}\right) \mu_{t}(d x)
$$

The following two results may be proved along the lines of Theorem 4.1 of [2] (in [2] the converse Kolmogorov inequality for Banach spaces is used; here we can use a similar inequality valid for the Hilbert space case due to Varadhan - see [6], Th. 3.3, Ch. VI).

LEMMA 2.2. Let $F$ be a closed subspace of $H$ and $q(x)=d(x, F)$. If $t>0$ and $\sigma_{t}=(1 / 2)\left(\mu_{t}+\bar{\mu}_{t}\right)$ then

$$
\int_{\{x: q(x) \leq r\}} q^{2}\left(\frac{1}{t}\right) d \mu_{t}<\left(\frac{9}{2}\right) r^{2}\left(1-12 \sigma_{t}^{[1 / t]+1}(\{x: q(x)>r\})\right)^{-1}
$$

holds for each $r>0$ such that $\sigma_{t}^{[1 / t]+1}(\{x: q(x)>r\})<1 / 12 .\left(\bar{\mu}_{t}\right.$ is the measure defined by $\bar{\mu}_{t}(B)=\mu_{t}(-B)$ for Borel sets $B ;[\cdot]$ denotes the integer part of a real number). 
THEOREM 2.3. $\left\{\nu_{t}: t>0\right\}$ is relatively (weakly) compact.

As in [6] (Ch. VI, Def. 2.3) we call $S$-operator a symmetric, positive, bounded operator $S$ on $H$ with finite trace, i.e. $\operatorname{tr}(S)=\sum_{j=1}^{\infty}\left\langle S e_{j}, e_{j}\right\rangle<\infty$ for some (every) orthonormal basis $\left\{e_{j}: j \in N\right\}$ of $H$. Recall that a class $\mathscr{A}$ of $S$-operators is compact ([6], Ch. VI, Def. 2.4) if: (I) $\sup _{S \in \mathscr{A}} \operatorname{tr}(S)<\infty$, (II) $\lim _{n \rightarrow \infty} \sup _{S \in \mathscr{A}} \sum_{j=n}^{\infty}\left\langle S e_{j}, e_{j}\right\rangle=0$ for some orthonormal basis $\left\{e_{j}\right\}$ of $H$.

Let $\left(L_{(1)}(H),\|\cdot\|_{1}\right)$ be the ideal, in the algebra of all operators on $H$, of trace class operators endowed with the trace norm and denote by $\mathscr{S}$ the class of all $S$-operators on $H$. The following proposition will be useful. We remark in passing that it proves in particular that the notion of compact class does not depend on the choice of orthonormal basis.

Proposition 2.4. Let $\mathscr{A} \subset \mathscr{S}$. Then $\mathscr{A}$ is a compact class if and only if $\mathscr{A}$ is relatively compact in $\left(L_{(1)}(H),\|\cdot\|_{1}\right)$.

Proof. For the sufficiency part, fix an (arbitrary) orthonormal basis $\left\{e_{j}: j \in N\right\}$ of $H$, define for each $n \in N$ the function $\psi_{n}: \mathscr{S} \rightarrow \mathbf{R}$ by $\psi_{n}(S)=\sum_{j=n}^{\infty}\left\langle S e_{j}, e_{j}\right\rangle$ and apply Dini's theorem.

Necessity. Let $\left\{e_{j}: j \in N\right\}$ be the orthonormal basis of $H$ for which both conditions of the definition of compact class hold. Take a sequence $\left\{S_{n}\right\} \subset \mathscr{A}$. We write $\|\cdot\|\left(\|\cdot\|_{2}\right)$ for the (Hilbert-Schmidt) operator norm.

Observe that for $S \in \mathscr{S}$ its symmetric positive square root $S^{1 / 2}$ is of Hilbert-Schmidt type and $\left\|S^{1 / 2}\right\|_{2}^{2}=\operatorname{tr}(S)$. The hypothesis implies that $\sup _{n}\left\|S_{n}^{1 / 2}\right\|_{2}<\infty$; hence by a standard procedure we may find a sequence $\left\{n_{k}\right\}$ and a bounded operator $T$ on $H$ such that $\left\{S_{n_{k}}^{1 / 2}\right\}$ converges to $T$ in the weak topology of operators.

Then $T$ is symmetric, positive and of Hilbert-Schmidt type:

$$
\begin{aligned}
\sum_{j=1}^{\infty}\left\|T e_{j}\right\|^{2} & =\sum_{j, i}\left\langle T e_{j}, e_{i}\right\rangle^{2} \leq \lim \inf _{k} \sum_{j, i}\left\langle S_{n_{k}}^{1 / 2} e_{j}, e_{l}\right\rangle^{2} \\
& =\lim \inf _{k}\left\|S_{n_{k}}^{1 / 2}\right\|_{2}^{2} \leq \sup _{S \in \mathscr{A}} \operatorname{tr}(S)<\infty .
\end{aligned}
$$

Next we prove that $S_{n_{k}}^{1 / 2} \rightarrow T$ strongly as $k \rightarrow \infty$. For $x \in H, m \in N$ we have

$$
\left\|\left(S_{n_{k}}^{1 / 2}-T\right) x\right\|^{2}=\sum_{j=1}^{m}\left\langle\left(S_{n_{k}}^{1 / 2}-T\right) x, e_{j}\right\rangle^{2}+\sum_{j=m+1}^{\infty}\left\langle x,\left(S_{n_{k}}^{1 / 2}-T\right) e_{j}\right\rangle^{2}
$$


since the last term is bounded by

$$
2\|x\|^{2} \sum_{j=m+1}^{\infty}\left(\left\langle S_{n_{k}} e_{j}, e_{j}\right\rangle+\left\|T e_{j}\right\|^{2}\right)
$$

the weak convergence of $\left\{S_{n_{k}}^{1 / 2}\right\}$ to $T$ and the compact class property imply that $S_{n_{k}}^{1 / 2} x \rightarrow T x$.

Having proved the strong convergence of $\left\{S_{n_{k}}\right\}$ to $T$, we can deduce from the inequality

$$
\left\|S_{n_{k}}^{1 / 2}-T\right\|_{2}^{2} \leq \sum_{j=1}^{m}\left\|\left(S_{n_{k}}^{1 / 2}-T\right) e_{j}\right\|^{2}+2 \sum_{j=m+1}^{\infty}\left(\left\langle S_{n_{k}} e_{j}, e_{j}\right\rangle+\left\|T e_{j}\right\|^{2}\right)
$$

that $\left\|S_{n_{k}}^{1 / 2}-T\right\|_{2} \rightarrow 0$ as $k \rightarrow \infty$. From this we obtain that $T^{2} \in L_{(1)}(H)$ and $\left\|S_{n_{k}}-T^{2}\right\|_{1} \rightarrow 0$, because

$$
\left\|S_{n_{k}}-T^{2}\right\|_{1} \leq\left\|S_{n_{k}}^{1 / 2}\right\|_{2} \cdot\left\|S_{n_{k}}^{1 / 2}-T\right\|_{2}+\left\|S_{n_{k}}^{1 / 2}-T\right\|_{2} \cdot\|T\|_{2} .
$$

We have thus proved that $\left\{S_{n}\right\}$ contains a convergent subsequence in $\left(L_{(1)}(H),\|\cdot\|_{1}\right)$.

REMARK. Let us recall that a probability measure $\gamma$ on a Banach space $E$ is Gaussian if it induces a normal (possibly degenerate) distribution on $\mathbf{R}$ via each $f \in E^{\prime}$. Consider the set $\Gamma$ of all Gaussian probability measures on $H$ with the topology of weak convergence and the set $\mathscr{S}$ with the topology induced by $\|\cdot\|_{1}$. Then the map which associates to every pair $(x, S)$ belonging to the topological product space $H \times \mathscr{S}$ the unique $\gamma \in \Gamma$ such that $\hat{\gamma}(y)=\exp \left(i\langle x, y\rangle-\frac{1}{2}\langle S y, y\rangle\right)$ is a homeomorphism (this follows from well-known facts - see [6], Ch. VI - together with Proposition 2.4).

After these preliminaries, we return to the family of operators $\left\{T_{t}\right\}$ associated to $\left\{\mu_{t}\right\}$.

TheOREM 2.5. $\left\{T_{t}: t>0\right\}$ is a compact class of $S$-operators.

Proof. Let $\left\{e_{J}: j \in N\right\}$ be an orthonormal basis of $H$. We must prove:

$$
\sup _{t>0} \operatorname{tr}\left(T_{t}\right)<\infty
$$

$$
\lim _{n \rightarrow \infty} \sup _{t>0} \sum_{j=n+1}^{\infty}\left\langle T_{t} e_{j}, e_{j}\right\rangle=0 \text {. }
$$

Claim (I) follows from Theorem 2.3 since

$$
\operatorname{tr}\left(T_{t}\right)=\int \frac{1}{1+\|x\|^{2}} \nu_{t}(d x) \leq \nu_{t}(H) .
$$


To prove (II) let $F_{n}$ be the subspace spanned by $\left\{e_{1}, \ldots, e_{n}\right\}$ and $q_{n}(x)=d\left(x, F_{n}\right)$. Observe that

$$
\lim _{n \rightarrow \infty} \sup _{t \in(0,1]} \sigma_{t}^{[1 / t]+1}\left(\left\{x: q_{n}(x)>r\right\}\right)=0,
$$

$\sigma_{t}$ being as in Lemma 2.2 and $r>0$. Putting $\lambda_{t}=\sigma_{t}^{[1 / t]+1}$, we obtain (1) from the relations

$$
\lambda_{t}\left(\left\{x: q_{n}(x)>r\right\}\right) \leq \lambda_{t}\left(\left\{x: q_{n}(x) \geq r\right\} \cap K\right)+\lambda_{t}\left(K^{c}\right)
$$

$(t \in(0,1])$, the relative compactness of $\left\{\lambda_{t}: t \in(0,1]\right\}$ (see the proof of Theorem 4.1 in [2]) and Dini's theorem (note that $q_{n} \downarrow 0$ pointwise).

Next, Lemma 2.2 and (1) yield: for every $r>0$ there exists $n_{0} \in N$ such that

$$
\sup _{t>0} \int_{\left\{x: q_{n}(x) \leq r\right\}} q_{n}^{2}\left(\frac{1}{t}\right) d \mu_{t} \leq 9 r^{2} \quad \text { for } n \geq n_{0} .
$$

This fact, Theorem 2.3, Dini's theorem and the inequalities

$$
\begin{aligned}
\sum_{j=n+1}^{\infty}\left\langle T_{t} e_{j}, e_{j}\right\rangle \leq & \int_{\left\{x: q_{n}(x) \leq r\right\}} q_{n}^{2}\left(\frac{1}{t}\right) d \mu_{t} \\
& +\int_{\left\{x: q_{n}(x) \geq r\right\} \cap K} q_{n}^{2}\left(\frac{1}{t}\right) d \mu_{t}+\nu_{t}\left(K^{c}\right)
\end{aligned}
$$

$(t>0)$ complete the proof through an easy argument.

Next, we define some auxiliary functions; the proofs of their properties are omitted (use Taylor's formula).

LEMMA 2.6. (a) Given $f \in C_{u}^{(2)}(H)$, define for every $x \in H$ $B_{f}(x, y)$

$$
=\left\{\begin{array}{l}
{\left[f(x+y)-f(x)-\frac{\left\langle f^{\prime}(x), y\right\rangle}{1+\|y\|^{2}}-\frac{1}{2} \frac{\left\langle f^{\prime \prime}(x) y, y\right\rangle}{\left(1+\|y\|^{2}\right)^{2}}\right] \frac{1+\|y\|^{2}}{\|y\|^{2}}, \quad y \neq 0,} \\
0, \quad y=0 .
\end{array}\right.
$$

Then the family $\left\{B_{f}(x, \cdot): x \in H\right\}$ is uniformly bounded and equicontinuous at each $y \in H$.

(b) For $y \in H$ let

$$
K(y, x)=\left\{\begin{array}{l}
{\left[\exp (i\langle x, y\rangle)-1-\frac{i\langle x, y\rangle}{1+\|x\|^{2}}\right] \frac{1+\|x\|^{2}}{\|x\|^{2}}, \quad x \neq 0,} \\
0, \quad x=0 .
\end{array}\right.
$$

Then $K(y, \cdot)$ is bounded and continuous on $H-\{0\}$. 
(c) For $y \in H$ let

$$
M(y, x)=K(y, x)+\frac{1}{2} \frac{\langle x, y\rangle^{2}}{\|x\|^{2}\left(1+\|x\|^{2}\right)}
$$

for $x \neq 0, M(y, 0)=0$. Then for every $r>0$ the family $\{M(y, \cdot):\|y\| \leq r\}$ is uniformly bounded and equicontinuous at each $x \in H$.

The proof of the next theorem requires the following uniqueness result (see [6], Ch. IV, Th. 8.1):

Lemma 2.7. If $x_{0}, x_{0}^{\prime} \in H, S, S^{\prime}$ are $S$-operators, $\nu, \nu^{\prime}$ are positive finite measures on $H$ such that $\nu(\{0\})=\nu^{\prime}(\{0\})=0$ and

$$
\begin{aligned}
& \exp \left[i\left\langle x_{0}, y\right\rangle-\frac{1}{2}\langle S y, y\rangle+\int K(y, \cdot) d \nu\right] \\
& \quad=\exp \left[i\left\langle x_{0}^{\prime}, y\right\rangle-\frac{1}{2}\left\langle S^{\prime} y, y\right\rangle+\int K(y, \cdot) d \nu^{\prime}\right]
\end{aligned}
$$

holds for all $y \in H$, then $x_{0}=x_{0}^{\prime}, S=S^{\prime}, \nu=\nu^{\prime}$ ( $K$ is as in (b) of the preceding lemma).

THEOREM 2.8. There exist $x_{0} \in H$, an $S$-operator $T_{0}$ and a positive finite measure $\nu_{0}$ on $H$ such that $x_{t} \rightarrow x_{0}, T_{t} \rightarrow T_{0}$ in $\|\cdot\|_{1}$ and $\nu_{t} \stackrel{w}{\rightarrow} \nu_{0}$ as $t \rightarrow 0$.

Proof. Given $t>0, y \in H$ and taking $M(y, \cdot)$ as in Lemma 2.6(c) we have

$$
\left(\frac{1}{t}\right)\left(\hat{\mu}_{t}(y)-1\right)=i\left\langle x_{t}, y\right\rangle-\left(\frac{1}{2}\right)\left\langle T_{t} y, y\right\rangle+\int M(y, \cdot) d \nu_{t} .
$$

Take a positive sequence $\left\{t_{n}\right\}$ such that $t_{n} \rightarrow 0$. Theorems 2.3, 2.5 and Proposition 2.4 imply that there exist a positive finite measure $\nu_{0}$ on $H$ and an $S$-operator $T_{0}$ such that

$$
T_{t_{n_{k}}} \rightarrow T_{0} \quad \text { in }\|\cdot\|_{1} \quad \text { and } \quad \nu_{t_{n_{k}}} \stackrel{w}{\rightarrow} \nu_{0} \quad(k \rightarrow \infty) .
$$

On the other hand, if $l: H \rightarrow \mathbf{C}$ is the sequentially $w^{*}$-continuous function such that $\hat{\mu}_{1}=\exp l$ and $l(0)=0$, the inequality

$$
\left|(1 / t)\left(\hat{\mu}_{t}(y)-1\right)-l(y)\right| \leq t \cdot \exp |l(y)|
$$

holds for $t \in(0,1]$, which shows that the left member of (1) converges to $l$ uniformly on the balls of $H$. Moreover $\left\{\int M(y, \cdot) d \nu_{t_{n_{k}}}\right\}$ converges in the 
same manner to $\int M(y, \cdot) d \nu_{0}$, by Lemma 2.6(c) and a well known result of R. Ranga Rao (see [6], Ch. II, Th. 6.8). The convergence of $\left\{\left\langle T_{t_{n_{k}}} y, y\right\rangle\right\}$ to $\left\langle T_{0} y, y\right\rangle$ is also uniform on the balls of $H$.

Then (1) gives that $\left\{\left\langle x_{t_{n_{k}}}, \cdot\right\rangle\right\}$ converges uniformly on the balls of $H$ and, consequently, there exists $x_{0} \in H$ such that $x_{t_{n_{k}}} \rightarrow x_{0}$ in $H$. Hence

$$
\hat{\mu}_{1}(y)=\exp \left[i\left\langle x_{0}, y\right\rangle-\left(\frac{1}{2}\right)\langle S y, y\rangle+\int K(y, \cdot) d\left(\nu_{0}-\nu_{0}(\{0\}) \delta_{0}\right)\right]
$$

for all $y \in H\left(K(y, \cdot)\right.$ is as in Lemma 2.6(b)) with $S=T_{0}-U, U$ being the symmetric operator defined by

$$
\langle U y, y\rangle=\int_{\{0\}^{c}} \frac{\langle x, y\rangle^{2}}{\|x\|^{2}\left(1+\|x\|^{2}\right)} \nu_{0}(d x) .
$$

We prove now that $S$ is an $S$-operator. Observing that $U$ is positive, it suffices to show that $S$ is positive; to this end, fix $y \in H$ and define $u$ : $H \rightarrow \mathbf{R}$ by

$$
u(x)= \begin{cases}\frac{\langle x, y\rangle^{2}}{\|x\|^{2}\left(1+\|x\|^{2}\right)}, & x \neq 0, \\ 0, & x=0 .\end{cases}
$$

This function is lower semicontinuous and bounded; then

$$
\begin{aligned}
\langle U y, y\rangle & =\int u d \nu_{0} \leq \lim \inf _{k} \int u d \nu_{t_{n_{k}}} \\
& =\lim \inf _{k}\left\langle T_{t_{n_{k}}} y, y\right\rangle=\left\langle T_{0} y, y\right\rangle
\end{aligned}
$$

since $\nu_{t_{n_{k}}} \stackrel{w}{\rightarrow} \nu_{0}$. This gives the positiveness of $S$.

Finally, we obtain the theorem by a standard argument involving subsequences, the uniqueness Lemma 2.7 and the following remark: if $\nu_{0}$ and $S$ are obtained as above (from a sequence $\left\{t_{n}\right\}$ ) then $\nu_{0}(\{0\})=\operatorname{tr}(S)$; in fact, putting $v(x)=\left(1+\|x\|^{2}\right)^{-1},(2)$ implies

$$
\begin{aligned}
\nu_{0}(\{0\}) & =\int v d \nu_{0}-\int_{\{0\}^{c}} v d \nu_{0}=\lim _{k} \int v d v_{t_{n_{k}}}-\operatorname{tr}(U) \\
& =\lim _{k} \operatorname{tr}\left(T_{t_{n_{k}}}\right)-\operatorname{tr}(U)=\operatorname{tr}\left(T_{0}\right)-\operatorname{tr}(U)=\operatorname{tr}(S) .
\end{aligned}
$$

COROLlaRY 2.9. (a) (Lévy-Khintchine representation) Let $\mu$ be a probability measure on $H$. Then $\mu$ is infinitely divisible if and only if there exist $x_{0} \in H$, an $S$-operator $S$ and a positive finite measure $\nu$ on $H$ such that 
$\nu(\{0\})=0$ which satisfy

$$
\hat{\mu}(y)=\exp \left[i\left\langle x_{0}, y\right\rangle-\frac{1}{2}\langle S y, y\rangle+\int K(y, \cdot) d \nu\right]
$$

for all $y \in H$.

The objects $x_{0}, S$ and $\nu$ are uniquely determined by $\mu$. (We will say that $\left[x_{0}, S, \nu\right]$ is the Lévy-Khintchine representation of $\mu$.)

(b) Given the semigroup $\left\{\mu_{t}: t \geq 0\right\}$, if $\left[x_{0}, S, \nu\right]$ is the Lévy-Khintchine representation of $\mu_{1}$, we have:

(i) $x_{t} \rightarrow x_{0}$;

(ii) $T_{t} \rightarrow S+U$ in $\|\cdot\|_{1}, U$ being the $S$-operator defined by

$$
\langle U y, y\rangle=\int_{\{0\}^{c}} \frac{\langle x, y\rangle^{2}}{\|x\|^{2}\left(1+\|x\|^{2}\right)} \nu(d x) ;
$$

(ii') if the $S$-operators $V_{t, \delta}$ are defined by

$$
\left\langle V_{t, \delta} y, y\right\rangle=\int_{\|x\| \leq \delta}\langle x, y\rangle^{2}\left(\frac{1}{t}\right) \mu_{t}(d x),
$$

then

$$
\lim _{\delta \rightarrow 0} \lim \sup _{t \rightarrow 0}\left\|V_{t, \delta}-S\right\|_{1}=0 ;
$$

(iii) $\nu_{t} \stackrel{w}{\rightarrow} \nu+\operatorname{tr}(S) \cdot \delta_{0}\left(\delta_{0}\right.$ is the unit point mass at 0$)$.

Proof of (ii'). Put $B_{\delta}=\{x \in H:\|x\| \leq \delta\}$. Let $T_{0}, \nu_{0}, U$ as in the proof of Theorem 2.8 and for $t>0, \delta>0$ consider the $S$-operators defined by:

$$
\begin{gathered}
\left\langle T_{t, \delta} y, y\right\rangle=\int_{B_{\delta}} \frac{\langle x, y\rangle^{2}}{\left(1+\|x\|^{2}\right)^{2}}\left(\frac{1}{t}\right) \mu_{t}(d x), \quad T_{t}^{\delta}=T_{t}-T_{t, \delta}, \\
\left\langle U_{\delta} y, y\right\rangle=\int_{B_{\delta}-\{0\}} \frac{\langle x, y\rangle^{2}}{\|x\|^{2}\left(1+\|x\|^{2}\right)^{2}} \nu_{0}(d x), \quad U^{\delta}=U-U_{\delta} .
\end{gathered}
$$

Fix $\delta>0$ such that $\nu_{0}(\{x:\|x\|=\delta\})=0$. Since $S=T_{0}-U$, for every $t>0$ we have

$$
V_{t, \delta}-S=\left(V_{t, \delta}-T_{t, \delta}\right)+\left(U-T_{t}^{\delta}\right)+\left(T_{t}-T_{0}\right)
$$

We know that $\lim _{t \rightarrow 0}\left\|T_{t}-T_{0}\right\|_{1}=0$.

For each $t>0$, since $V_{t, \delta}-T_{t, \delta}$ is positive, one has

$$
\left\|V_{t, \delta}-T_{t, \delta}\right\|_{1}=\operatorname{tr}\left(V_{t, \delta}-T_{t, \delta}\right)=\int_{B_{\delta}}\|x\|^{2}\left(\frac{2+\|x\|^{2}}{1+\|x\|^{2}}\right) \nu_{t}(d x) ;
$$


hence the choice made of $\delta$ gives

$$
\lim _{t \rightarrow 0}\left\|V_{t, \delta}-T_{t, \delta}\right\|_{1}=\int_{B_{\delta}}\|x\|^{2}\left(\frac{2+\|x\|^{2}}{1+\|x\|^{2}}\right) \nu_{0}(d x) .
$$

For the remaining term, observe first that $\left\{T_{t}^{\delta}: t>0\right\}$ is relatively $\|\cdot\|_{1}$-compact. Next, consider a $\|\cdot\|_{1}$-limit $T_{0}^{\delta}$ of a sequence $\left\{T_{t_{n}}^{\delta}\right\}$ with $t_{n} \rightarrow 0$; again by the choice of $\delta$,

$$
\left\langle T_{0}^{\delta} y, y\right\rangle=\lim _{n \rightarrow \infty} \int_{B_{\delta}^{c}} \frac{\langle x, y\rangle^{2}}{\|x\|^{2}\left(1+\|x\|^{2}\right)} \nu_{t_{n}}(d x)=\left\langle U^{\delta} y, y\right\rangle .
$$

This shows that $T_{t}^{\delta} \rightarrow U^{\delta}$ in $\|\cdot\|_{1}$ as $t \rightarrow 0$. Then

$$
\lim _{t \rightarrow 0}\left\|U-T_{t}^{\delta}\right\|_{1}=\left\|U_{\delta}\right\|_{1} \text {. }
$$

The preceding argument proves that if $\delta>0$ satisfies

$$
\nu_{0}(\{x:\|x\|=\delta\})=0
$$

one has

$$
\begin{aligned}
\lim \sup _{t \rightarrow 0}\left\|V_{t, \delta}-S\right\|_{1} \leq & \int_{B_{\delta}}\|x\|^{2}\left(\frac{2+\|x\|^{2}}{1+\|x\|^{2}}\right) \nu_{0}(d x) \\
& +\int_{B_{\delta}-\{0\}} \frac{1}{1+\|x\|^{2}} \nu_{0}(d x) .
\end{aligned}
$$

The finiteness of $\nu_{0}$ implies the result.

Remark. Part (a) is due to Varadhan (see [6]). (b) may be compared with the statement of the Lévy-Khintchine representation in Banach spaces in [1] (Corollary 1.11); in particular, (ii') strengthens the limit formula (2) in [1].

3. Semigroups of convolution operators. Given a probability measure $\mu$ on $H$, we define the convolution operator $P_{\mu}$ on $C_{u}(H)$ by $P_{\mu} f(x)=$ $\int f(x+y) \mu(d y)\left(f \in C_{u}(H), x \in H\right)$ and to every semigroup of measures $\left\{\mu_{t}: t \geq 0\right\}$ on $H$ we associate the family $\left\{P_{t}: t \geq 0\right\}$ such that $P_{t}=P_{\mu_{t}}$ for each $t$. It is a strongly continuous semigroup of operators; in other words: (1) $P_{0}=I$ (the identity operator), (2) $P_{s} P_{t}=P_{s+t}$ for $s \geq 0$, $t \geq 0$, and (3) $\lim _{t \rightarrow 0}\left\|P_{t} f-f\right\|=0$ for each $f \in C_{u}(H)$. Let us remark that every strongly continuous semigroup of convolution operators on $C_{u}(H)$ may be obtained from a semigroup of measures as above.

Given such a semigroup $\left\{P_{t}: t \geq 0\right\}$ define $A_{t}=(1 / t)\left(P_{t}-I\right)$ for $t>0$ and the operator $A$ by $A f=\lim _{t \rightarrow 0} A_{t} f$ on the linear subspace $D$ of 
those $f \in C_{u}(H)$ for which the limit exists in $C_{u}(H) . A$ is called the infinitesimal generator of $\left\{P_{t}\right\}$ and $D$ its domain. Throughout this section $\left\{\mu_{t}\right\},\left\{P_{t}\right\}, D$ and $A$ are related in this manner and $\left[x_{0}, S, \nu\right]$ is the Lévy-Khintchine representation of $\mu_{1}$.

THEOREM 3.1. $C_{u}^{(2)}(H) \subset D$ and for every $f \in C_{u}^{(2)}(H)$ and each $x \in H$

$$
\begin{aligned}
A f(x)= & \left\langle x_{0}, f^{\prime}(x)\right\rangle+\frac{1}{2} \operatorname{tr}\left(S f^{\prime \prime}(x)\right) \\
& +\int\left[f(x+y)-f(x)-\frac{\left\langle f^{\prime}(x), y\right\rangle}{1+\|y\|^{2}}\right] \frac{1+\|y\|^{2}}{\|y\|^{2}} \nu(d y) .
\end{aligned}
$$

Proof. Fix $f \in C_{u}^{(2)}(H)$; for $t>0$ and $x \in H$ we have

$$
\begin{aligned}
A_{t} f(x)= & \left\langle x_{t}, f^{\prime}(x)\right\rangle+\frac{1}{2} \int \frac{\left\langle f^{\prime \prime}(x) y, y\right\rangle}{\left(1+\|y\|^{2}\right)^{2}}\left(\frac{1}{t}\right) \mu_{t}(d y) \\
& +\int B_{f}(x, \cdot) d \nu_{t}
\end{aligned}
$$

( $B_{f}$ as in Lemma 2.6(a)).

First, we prove that for every symmetric operator $\Delta$ on $H$

$$
\int \frac{\langle\Delta y, y\rangle}{\left(1+\|y\|^{2}\right)^{2}}\left(\frac{1}{t}\right) \mu_{t}(d y)=\operatorname{tr}\left(T_{t} \Delta\right) .
$$

If $\Delta$ is an orthogonal projection $P$, taking orthonormal bases $\left\{e_{i}\right.$ : $i \in I\}$ and $\left\{e_{j}: j \in J\right\}$ of $P(H)$ and $P(H)^{\perp}$, respectively (with $I \cap J=$ $\varnothing$ ), one has: $\left\{e_{i}: i \in I \cup J\right\}$ is an orthonormal basis of $H$ and for every $y \in H$

$$
\begin{aligned}
& \left\langle P y, e_{i}\right\rangle= \begin{cases}\left\langle y, e_{i}\right\rangle, & i \in I, \\
0, & i \in J,\end{cases} \\
& \langle P y, y\rangle=\|P y\|^{2}=\sum_{i \in I \cup J}\left\langle y, P e_{i}\right\rangle\left\langle y, e_{i}\right\rangle .
\end{aligned}
$$

Hence

$$
\int \frac{\langle P y, y\rangle}{\left(1+\|y\|^{2}\right)^{2}}\left(\frac{1}{t}\right) \mu_{t}(d y)=\sum_{i \in I \cup J}\left\langle T_{t} P e_{i}, e_{i}\right\rangle=\operatorname{tr}\left(T_{t} P\right) .
$$

Given a symmetric operator $\Delta$, the spectral theorem implies that there exists a sequence $\left\{\Delta_{n}\right\}$ of operators which are finite linear combinations 
of orthogonal projections such that $\left\|\Delta_{n}-\Delta\right\| \rightarrow 0(n \rightarrow \infty)$. Since (2) is true for each $\Delta_{n}$, we may apply Lebesgue's dominated convergence theorem to the integrals and the inequality $\left|\operatorname{tr}\left(T_{t} \Delta_{n}\right)-\operatorname{tr}\left(T_{t} \Delta\right)\right| \leq\left\|T_{t}\right\|_{1}$. $\left\|\Delta_{n}-\Delta\right\|$ in order to conclude that (2) holds for $\Delta$.

Consequently, we can rewrite (1) as

$$
A_{t} f(x)=\left\langle x_{t}, f^{\prime}(x)\right\rangle+\frac{1}{2} \operatorname{tr}\left(T_{t} f^{\prime \prime}(x)\right)+\int B_{f}(x, \cdot) d \nu_{t} .
$$

Let $T_{0}=S+U(U$ as in Corollary $2.9(\mathrm{~b}))$ and $\nu_{0}=\nu+\operatorname{tr}(S) \cdot \delta_{0}$. For every $t>0$ and $x \in H$ we have

$$
\begin{gathered}
\left\langle x_{t}, f^{\prime}(x)\right\rangle-\left\langle x_{0}, f^{\prime}(x)\right\rangle \mid \leq\left\|x_{t}-x_{0}\right\| \cdot\left\|f^{\prime}\right\|, \\
\left|\operatorname{tr}\left(T_{t} f^{\prime \prime}(x)\right)-\operatorname{tr}\left(T_{0} f^{\prime \prime}(x)\right)\right| \leq\left\|T_{t}-T_{0}\right\|_{1} \cdot\left\|f^{\prime \prime}\right\| ;
\end{gathered}
$$

moreover $\left\{B_{f}(x, \cdot): x \in H\right\}$ is uniformly bounded and equicontinuous (Lemma 2.6 (a)). Then Corollary 2.9(b) and the result cited in the proof of Theorem 2.8 imply that

$$
A_{t} f(x) \rightarrow\left\langle x_{0}, f^{\prime}(x)\right\rangle+\frac{1}{2} \operatorname{tr}\left(T_{0} f^{\prime \prime}(x)\right)+\int B_{f}(x, \cdot) d \nu_{0}
$$

uniformly in $x \in H$ as $t \rightarrow 0$. This shows that $f \in D$.

But

$$
\begin{aligned}
\int B_{f}(x, \cdot) d \nu_{0}= & \int\left[f(x+y)-f(x)-\frac{\left\langle f^{\prime}(x), y\right\rangle}{1+\|y\|^{2}}\right] \frac{1+\|y\|^{2}}{\|y\|^{2}} \nu(d y) \\
& -\frac{1}{2} \int \frac{\left\langle f^{\prime \prime}(x) y, y\right\rangle}{\|y\|^{2}\left(1+\|y\|^{2}\right)^{2}} \nu(d y)
\end{aligned}
$$

and the last integral equals $\operatorname{tr}\left(U f^{\prime \prime}(x)\right)$ (argue as in the proof of (2)). This gives the announced expression for $A f$.

Let us mention now without proof two corollaries of Theorem 3.1 which are generalizations of results in [3] (Lemmas 8 and 6).

Corollary 3.2. $\left\{P_{t}: t \geq 0\right\}$ is uniformly continuous on $C_{u}(H)$ (i.e. $P_{t}$ tends to $I$ in the operator norm as $t \rightarrow 0$ ) if and only if there exists a positive finite measure $\lambda$ on $H$ such that $\mu_{t}=\exp \left[t\left(\lambda-\|\lambda\| \delta_{0}\right)\right]$. (For a finite measure $\alpha, \exp (\alpha)$ is defined by the usual expansion, converging in the total variation norm).

COROLlARY 3.3. The following conditions are equivalent:

(1) $A$ is of local character on $D$ (i.e. Af $(x)=0$ when $f \in D$ vanishes in a neighborhood of $x \in H$ ). 
(2) $\mu_{1}$ is Gaussian.

(3) $\lim _{t \rightarrow 0}(1 / t) \mu_{t}\left(V^{c}\right)=0$ for every neighborhood $V$ of 0 .

REMARK. Theorem 3.1 includes results of Courrège for the finite-dimensional case ([3], Théorèmes 1 and 2). In that situation $C_{u}^{(2)}(H)$ is dense in $C_{u}(H)$, but this is no longer true when $H$ has infinite dimension. This fact was proved by $\mathrm{D}$. Herrero (personal communication) who, using arguments of [7] $\left(\$ 5\right.$, Th. 1), showed that $C_{u}^{(2)}\left(l^{2}\right)$ is not dense in $C_{u}\left(l^{2}\right)$ (explicitly: if $A=\left\{x \in l^{2}: x_{l} \leq 0,\|x\| \leq 1\right\}$ and $f(x)=\min \{1, d(x, A)\}$ then for all $g \in C_{u}^{(2)}\left(l^{2}\right)$ one has $\left.\|f-g\| \geq 1 / 2\right){ }^{1}$

In view of this negative result it seems of interest to show that for a special class of semigroups - namely, semigroups without Gaussian part - there exists a dense subspace of $C_{u}(H)$ which is contained in the domain of every generator. Again, the density result depends on [7].

Let us call $C_{u, L}^{(1)}(H)$ the space of those functions $f \in C_{u}(H)$ which are Fréchet differentiable with a derivative $f^{\prime}$ that satisfies: (i) $\left\|f^{\prime}\right\|=$ $\sup _{x \in H}\left\|f^{\prime}(x)\right\|<\infty$, (ii) for some $\delta>0$ and $M>0$ it holds $\| f^{\prime}(x)$ $f^{\prime}(y)\|\leq M\| x-y \|$ when $\|x-y\| \leq \delta$.

Proposition 3.4. $C_{u, L}^{(1)}(H)$ is dense in $C_{u}(H)$.

Proof. It is proved in [7], $\$ 4$, Corollary 4 that given closed subsets $A, B$ of $H$ at positive distance, there exists a continuous function $f$ : $H \rightarrow[0,1]$ with Lipschitz derivative such that $\left.f\right|_{A}=0$ and $\left.f\right|_{B}=1$. For such a pair of sets with $d(A, B)=4 \delta>0$ we may prove, applying the result to $A^{\prime}=\{x: d(x, A) \leq \delta\}$ and $B^{\prime}=\{x: d(x, A) \geq 2 \delta\}$, that in fact $f$ can be chosen with $\left\|f^{\prime}\right\|<\infty$ and then $f \in C_{u, L}^{(1)}(H)$.

The proof is completed by using the following modification of a well known fact whose proof we omit (see [5], Ch. 7, Problem P): let $M$ be a metric space and $L$ a linear subspace of $C_{u}(M)$. Suppose that for each pair of closed subsets $A, B$ of $M$ at positive distance and for each real interval $[a, b]$ there exists $f \in L$ such that $a \leq f \leq b,\left.f\right|_{A}=a$ and $\left.f\right|_{B}=b$. Then $L$ is dense in $C_{u}(M)$.

LEMMA 3.5. Let $f \in C_{u, L}^{(1)}(H)$ and define

$$
L_{f}(x, y)=\left\{\begin{array}{l}
{\left[f(x+y)-f(x)-\frac{\left\langle f^{\prime}(x), y\right\rangle}{1+\|y\|^{2}}\right] \frac{1+\|y\|^{2}}{\|y\|^{2}}, \quad y \neq 0,} \\
0, \quad y=0 .
\end{array}\right.
$$

Then $\left\{L_{f}(x, \cdot): x \in H\right\}$ is equicontinuous at every $y \neq 0$ and uniformly bounded.

\footnotetext{
${ }^{1}$ We thank D. Herrero for many helpful conversations on this point and J. Eells, who suggested that [7] might be relevant in a letter to A. de Acosta.
} 
THEOREM 3.6. Suppose that $S=0$. Then $C_{u, L}^{(1)}(H) \subset D$ and for every $f \in C_{u, L}^{(1)}(H)$ and each $x \in H$

$$
\begin{aligned}
A f(x)= & \left\langle x_{0}, f^{\prime}(x)\right\rangle \\
& +\int\left[f(x+y)-f(x)-\frac{\left\langle f^{\prime}(x), y\right\rangle}{1+\|y\|^{2}}\right] \frac{1+\|y\|^{2}}{\|y\|^{2}} \nu(d y) .
\end{aligned}
$$

Proof. Let $f \in C_{u, L}^{(1)}(H)$; for $t>0$ and $x \in H$ one has

$$
A_{t} f(x)=\left\langle x_{t}, f^{\prime}(x)\right\rangle+\int L_{f}(x, \cdot) d \nu_{t}
$$

( $L_{f}$ as in Lemma 3.5).

By the preceding lemma and the fact that $\nu_{t} \stackrel{w}{\rightarrow} \nu$ and $\nu(\{0\})=0$ (Corollary 2.9(b)) we may deduce that $\int L_{f}(x, \cdot) d \nu_{t} \rightarrow \int L_{f}(x, \cdot) d \nu$ uniformly in $x$ as $t \rightarrow 0$, which gives the theorem. The proof requires a slight generalization of a result previously used: let $M$ be a complete separable metric space and $\left\{\mu_{\alpha}\right\}$ a relatively compact net of positive finite measures on (the Borel sets of) $M$, weakly convergent to $\mu$. Then if $\mathscr{F}$ is a set of Borel functions from $M$ to $\mathbf{R}$ which is uniformly bounded and equicontinuous at each $x \in \Lambda^{c}, \Lambda$ being a closed set with $\mu(\Lambda)=0$, one has

$$
\lim _{\alpha} \sup _{f \in \mathscr{F}}\left|\int f d \mu_{\alpha}-\int f d \mu\right|=0
$$

For our final statement, let us recall the construction of the Hilbert space of a centered Gaussian measure $\gamma$ on a separable Banach space $E$ (see [1], §5). Let $L^{2}(\gamma)$ be the Hilbert space of (classes of) square $\gamma$-integrable functions on $E$ and $\widehat{E^{\prime}}$ be the closure in $L^{2}(\gamma)$ of $E^{\prime}$ endowed with the $L^{2}(\gamma)$-norm, denoted $\|\cdot\|_{L^{2}}=\langle\cdot, \cdot\rangle_{L^{2}}^{1 / 2}$. Define $H_{\gamma}$ as the linear subspace of $E$ of those $h$ such that $f \mapsto f(h)$ is $L^{2}$-continuous on $E^{\prime}$. For $h \in H_{\gamma}$ the Riesz representation gives a unique $\phi(h) \in \widehat{E^{\prime}}$ such that $f(h)=\langle\phi(h), f\rangle_{L^{2}}$ for all $f \in E^{\prime}$; it is verified that $\phi: H_{\gamma} \rightarrow \widehat{E^{\prime}}$ is an algebraic isomorphism and $H_{\gamma}$ becomes a Hilbert space isomorphic to $\widehat{E^{\prime}}$ by defining $\langle h, k\rangle_{\gamma}=\langle\phi(h), \phi(k)\rangle_{L^{2}}\left(h, k, \in H_{\gamma}\right)$. The norm $\|\cdot\|_{\gamma}$ is stronger than $\|\cdot\|$ (the norm of $E$ ) on $H_{\gamma}$ and $\vec{H}_{\gamma}^{\|\cdot\|}$ coincides with the support of $\gamma$.

When $E=H$ is a Hilbert space with inner product $\langle\cdot, \cdot\rangle$, one has $\left\langle\langle\cdot, y\rangle,\left\langle\cdot, y^{\prime}\right\rangle\right\rangle_{L^{2}}=\left\langle S y, y^{\prime}\right\rangle\left(y, y^{\prime}, \in H\right)$, where $S$ is the covariance operator of $\gamma ; h \in H_{\gamma}$ iff $y \mapsto\langle x, y\rangle$ is continuous on $H$ with respect to 
the bilinear form given by $S$ and $\phi: H_{\gamma} \rightarrow \widehat{H^{\prime}}$ is characterized by $\langle h, y\rangle=$ $\langle\phi(h),\langle\cdot, y\rangle\rangle_{L^{2}}$ for $h \in H_{\gamma}, y \in H$. Moreover $S(H)=\phi^{-1}\left(H^{\prime}\right)$ and $\left\langle S x, S x^{\prime}\right\rangle_{\gamma}=\left\langle S^{1 / 2} x, S^{1 / 2} x^{\prime}\right\rangle$ for $x, x^{\prime}, \in H$; by the density of $S(H)$ in $H_{\gamma}$ it follows that

$$
\langle S x, h\rangle_{\gamma}=\langle x, h\rangle \text { for } x \in H, h \in H_{\gamma} .
$$

Given a real function $f$ on the Banach space $E$, we say (as in [4]) that $f$ is twice $H_{\gamma}$-differentiable at $x \in E$ if the function $g(h)=f(x+h), g$ : $H_{\gamma} \rightarrow \mathbf{R}$ is twice $\|\cdot\|_{\gamma}$-Fréchet differentiable at 0 ; in this case, we denote by $D^{2} f(x)$ the operator on $H_{\gamma}$ associated with the second derivative of $g$ at 0 . This notion is weaker than $\|\cdot\|$-Fréchet differentiability. If $T \in$ $L_{(1)}\left(H_{\gamma}\right)$ we denote its trace by $\operatorname{tr}_{\gamma}(T)$.

Now we can extend in the Hilbert space case Corollary 3.2 of [4].

COROLlaRY 3.7. Let $\gamma$ be the centered Gaussian measure with covariance operator $S$. The class of functions $f$ which satisfy the following conditions is dense in $C_{u}(H)$.

(i) $f \in C_{u, L}^{(1)}(H)$,

(ii) for each $x \in H, f$ is twice $H_{\gamma}$-differentiable at $x$ and $D^{2} f(x) \in$ $L_{(1)}\left(H_{\gamma}\right)$,

(iii) $D^{2} f: H \rightarrow L_{(1)}\left(H_{\gamma}\right)$ is bounded and uniformly continuous,

(iv) $f \in D$ and

$$
\begin{aligned}
A f(x)= & \left\langle x_{0}, f^{\prime}(x)\right\rangle+\frac{1}{2} \operatorname{tr}_{\gamma}\left(D^{2} f(x)\right) \\
& +\int\left[f(x+y)-f(x)-\frac{\left\langle f^{\prime}(x), y\right\rangle}{1+\|y\|^{2}}\right] \frac{1+\|y\|^{2}}{\|y\|^{2}} \nu(d y)
\end{aligned}
$$

for every $x \in H$.

Proof. The proof of Corollary 3.2 in [4] and Theorem 3.6 of the present paper show that the set of functions $f(x)=\int_{0}^{\infty} e^{-t}\left(\Gamma_{t} g\right)(x) d t$, with $g \in C_{u, L}^{(1)}(H)$ and $\left\{\Gamma_{t}\right\}$ the semigroup of operators associated to $\gamma$, is dense in $C_{u}(H)$ and satisfies properties (i)-(iv) (the results of [4] needed are proved there only when $H_{\gamma}$ is dense in $H$, but the proofs remain valid in general).

REMARK. The class of functions defined in Corollary 3.7 contains $C_{u}^{(2)}(H)$ and the expression for $A f$ coincides with that of Theorem 3.1. In fact, the following statement holds: if $f: H \rightarrow \mathbf{R}$ is a continuous function with second $\|\cdot\|$-Fréchet derivative $f^{\prime \prime}(x)$ at some point $x \in H$ then $f$ is 
twice $H_{\gamma}$-differentiable at $x, D^{2} f(x)=\left.S f^{\prime \prime}(x)\right|_{H_{\gamma}} \in L_{(1)}\left(H_{\gamma}\right)$ and $\operatorname{tr}_{\gamma}\left(D^{2} f(x)\right)=\operatorname{tr}\left(S f^{\prime \prime}(x)\right)$.

To prove this, observe that we have the maps

$$
H_{\gamma} \stackrel{\iota}{\rightarrow} H, \quad H \stackrel{\sigma}{\rightarrow} H^{\prime} \stackrel{\kappa}{\rightarrow}\left(H_{\gamma}\right)^{\prime} \stackrel{\tau^{-1}}{\rightarrow} H_{\gamma},
$$

$\sigma$ and $\tau$ being the Riesz representations for $H$ and $H_{\gamma}, \iota$ the inclusion map, which satisfies $\|\iota(x)\| \leq c\|x\|_{\gamma}$ with $c^{2}=\int\|y\|^{2} \gamma(d y), \kappa$ the injection which to every $\psi \in H^{\prime}$ associates its restriction to $H_{\gamma}$. Using $(*)$ we may check that $\tau^{-1} \kappa \sigma=S$.

If $f$ is twice $\|\cdot\|$-Fréchet differentiable at $x \in H$ then it is twice $H_{\gamma}$-differentiable at $x$ and in fact $D^{2} f(x)=\tau^{-1} \kappa \sigma f^{\prime \prime}(x) \iota=S f^{\prime \prime}(x) \iota$. Take now an orthonormal basis $\left\{e_{n}: n \in N\right\}$ of $H$ such that $S e_{n}=\lambda_{n} e_{n}$ with $\lambda_{n} \geq 0$ and put $u_{n}=\lambda_{n}^{(1 / 2)} e_{n}$ for $n \in N$ such that $\lambda_{n}>0 ;(*)$ gives that $\left\{u_{n}: n \in N\right.$ with $\left.\lambda_{n}>0\right\}$ is an orthonormal basis of $H_{\gamma}$. In order to conclude that $D^{2} f(x)$ is of trace class on $H_{\gamma}$ note that $S: H \rightarrow H_{\gamma}$ is the (Hilbertian) adjoint of $\iota: H_{\gamma} \rightarrow H$, which is of Hilbert-Schmidt type (this is well known: $\left.\left\|\iota\left(u_{n}\right)\right\|^{2}=\lambda_{n}\right)$.

To complete the proof, observe that by $(*)$ we have

$$
\begin{aligned}
\left\langle D^{2} f(x) u_{n}, u_{n}\right\rangle_{\gamma} & =\left\langle f^{\prime \prime}(x) \iota u_{n}, u_{n}\right\rangle=\lambda_{n}\left\langle f^{\prime \prime}(x) e_{n}, e_{n}\right\rangle \\
& =\left\langle S f^{\prime \prime}(x) e_{n}, e_{n}\right\rangle
\end{aligned}
$$

for every $n \in N$ such that $\lambda_{n}>0$.

Thus we have for each semigroup $\left\{P_{t}\right\}$ a dense subspace of $C_{u}(H)$, depending on the Gaussian part of $\mu_{1}$, on which we can describe the infinitesimal generator of $\left\{P_{t}\right\}$. It is not known to us if in the infinite-dimensional case it is true that the intersection of the domains of the generators of all such semigroups is dense in $C_{u}(H)$ (the finite-dimensional case is solved by the cited result of Courrège, i.e. Theorem 3.1 for $H=\mathbf{R}^{n}$ ). In view of Theorem 3.6, it seems that the Gaussian case must provide the answer.

Acknowledgment. I wish to express my gratitude to Professor Alejandro de Acosta, who directed this work, for his generous guidance.

\section{REFERENCES}

[1] A. de Acosta, A. Araujo and E. Giné, On Poisson measures, Gaussian measures and the central limit theorem in Banach spaces, Advances in Probability, 4, 1-68. New York: M. Dekker 1978

[2] A. de Acosta and J. Samur, Infinitely divisible probability measures and the converse Kolmogorov inequality in Banach spaces, Studia Math., 66 (1979), 143-160. 
[3] Ph. Courrège, Génerateur infinitésimal d'un semigroupe de convolution sur $R^{n}$ et formule de Lévy-Khintchine, Bull. Sci. Math., $882^{\text {ème }}$ série, (1964), 3-30.

[4] L. Gross, Potential theory on Hilbert space, J. Functional Analysis, 1 N², (1967), 123-181.

[5] J. Kelley, General Topology, Princeton: Van Nostrand 1955

[6] K. R. Parthasarathy, Probability Measures on Metric Spaces, New York: Academic Press 1967

[7] J. Wells, Differentiable functions on Banach spaces with Lipschitz derivatives, J. Differential Geometry, 8 (1973), 135-152.

[*] K. Sundaresan, Geometry and nonlinear analysis in Banach spaces, Pacific J. Math., $102 \mathrm{~N}^{\circ} 2$, (1982).

Received March 23, 1983. This work was done at the Instituto Venezolano de Investigaciones Cientificas, Venezuela and was partially supported by a fellowship of CORDIPLAN and CONICIT Grant 51-26.S1.0893, Venezuela.

DEPARTAMENTO DE MATEMÁtica

Universidad Nacional de La Plata

CaSilla de CoRreo 172

1900 La Plata

ARgENTINA 



\title{
PACIFIC JOURNAL OF MATHEMATICS EDITORS
}

\author{
Donald BABBITt (Managing Editor) \\ University of California \\ Los Angeles, CA 90024 \\ J. DugunduI \\ University of Southern California \\ Los Angeles, CA 90089-1113 \\ R. FINN \\ Stanford University \\ Stanford, CA 94305 \\ HermanN FlaschKa \\ University of Arizona \\ Tucson, AŻ 85721
}

C. C. Moore

University of California

Berkeley, CA 94720

ARTHUR Ogus

University of California

Berkeley, CA 94720

Hugo Rossi

University of Utah

Salt Lake City, UT 84112

H. SAMELSON

Stanford University

Stanford, CA 94305

ASSOCIATE EDITORS
R. ARENS
E. F. BECKENBACH
B. H. NEUMANN
F. WOLF
K. YoSHIDA (1906-1982)

\section{SUPPORTING INSTITUTIONS}

$\begin{array}{ll}\text { UNIVERSITY OF ARIZONA } & \text { UNIVERSITY OF OREGON } \\ \text { UNIVERSITY OF BRITISH COLUMBIA } & \text { UNIVERSITY OF SOUTHERN CALIFORNIA } \\ \text { CALIFORNIA INSTITUTE OF TECHNOLOGY } & \text { STANFORD UNIVERSITY } \\ \text { UNIVERSITY OF CALIFORNIA } & \text { UNIVERSITY OF HAWAII } \\ \text { MONTANA STATE UNIVERSITY } & \text { UNIVERSITY OF TOKYO } \\ \text { UNIVERSITY OF NEVADA, RENO } & \text { UNIVERSITY OF UTAH } \\ \text { NEW MEXICO STATE UNIVERSITY } & \text { WASHINGTON STATE UNIVERSITY } \\ \text { OREGON STATE UNIVERSITY } & \text { UNIVERSITY OF WASHINGTON }\end{array}$

The Supporting Institutions listed above contribute to the cost of publication of this Journal, but they are not owners or publishers and have no responsibility for its content or policies.

Mathematical papers intended for publication in the Pacific Journal of Mathematics should be in typed form or offset-reproduced (not dittoed), double spaced with large margins. Please do not use built up fractions in the text of the manuscript. However, you may use them in the displayed equations. Underline Greek letters in red, German in green, and script in blue. The first paragraph must be capable of being used separately as a synopsis of the entire paper. In particular it should contain no bibliographic references. Please propose a heading for the odd numbered pages of less than 35 characters. Manuscripts, in triplicate, may be sent to any one of the editors. Please classify according to the scheme of Math. Reviews, Index to Vol. 39. Supply name and address of author to whom proofs should be sent. All other communications should be addressed to the managing editor, or Elaine Barth, University of California, Los Angeles, California 90024.

There are page-charges associated with articles appearing in the Pacific Journal of Mathematics. These charges are expected to be paid by the author's University, Government Agency or Company. If the author or authors do not have access to such Institutional support these charges are waived. Single authors will receive 50 free reprints; joint authors will receive a total of 100 free reprints. Additional copies may be obtained at cost in multiples of 50 .

The Pacific Journal of Mathematics is issued monthly as of January 1966. Regular subscription rate: $\$ 190.00$ a year (5 Vols., 10 issues). Special rate: $\$ 66.00$ a year to individual members of supporting institutions.

Subscriptions, orders for numbers issued in the last three calendar years, and changes of address should be sent to Pacific Journal of Mathematics, P.O. Box 969, Carmel Valley, CA 93924, U.S.A. Old back numbers obtainable from Kraus Periodicals Co., Route 100, Millwood, NY 10546.

The Pacific Journal of Mathematics at P.O. Box 969, Carmel Valley, CA 93924 (ISSN 0030-8730) publishes 5 volumes per year. Application to mail at Second-class postage rates is pending at Carmel Valley, California, and additional mailing offices. Postmaster: Send address changes to Pacific Journal of Mathematics, P.O. Box 969, Carmel Valley, CA 93924.

PUBLISHED BY PACIFIC JOURNAL OF MATHEMATICS, A NON-PROFIT CORPORATION

Copyright $@ 1984$ by Pacific Journal of Mathematics 


\section{Pacific Journal of Mathematics}

\section{Vol. 115, No. $2 \quad$ October, 1984}

Ersan Akyildiz, Gysin homomorphism and Schubert calculus ...........257

Marilyn Breen, Clear visibility and unions of two starshaped sets in the

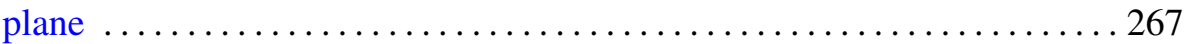

Robert F. Brown, Retraction methods in Nielsen fixed point theory . ......277

Herbert Busemann and Bhalchandra B. Phadke, A general version of Beltrami's theorem in the large ............................... 299

Gerald Arthur Edgar and Robert Francis Wheeler, Topological properties of Banach spaces ............................... 317

Yaakov Friedman and Bernard Russo, Conditional expectation without

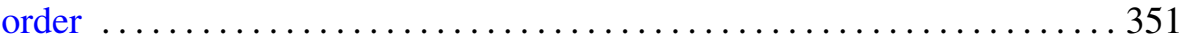

Robert Allen Goggins, Cobordism of manifolds with strong almost tangent

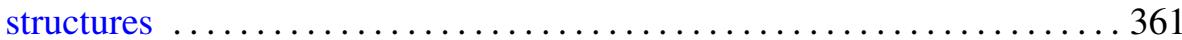

Mike Hoffman, Noncoincidence index of manifolds . . . . . . . . . . . . . 373

William H. Julian, $\varepsilon$-continuity and monotone operations $\ldots \ldots \ldots \ldots 385$

Gerasimos E. Ladas, Y. G. Sficas and I. P. Stavroulakis, Nonoscillatory

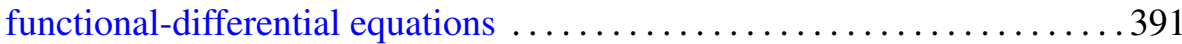

Arnold William Miller and Karel Libor Prikry, When the continuum has

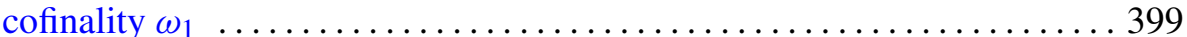

Jean-Leah Mohrherr, Density of a final segment of the truth-table



Carl Norman Mutchler, The flat Cauchy problem for radially hyperbolic operators from a characteristic manifold of high codimension ...

Kenji Nakagawa, On the orders of automorphisms of a closed Riemann surface

W. Ricker, Representation of vector-valued functions by Laplace transforms

Jorge Donato Samur, On semigroups of convolution operators in Hilbert

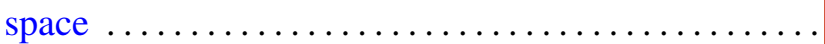

Joseph Gail Stampfli, One-dimensional perturbations of operators 481 Andrew George Earnest and John Sollion Hsia, Correction to: "Spinor norms of local integral rotations. II" 